\title{
A dinâmica de interação institutos de pesquisas/empresas: os condicionantes pró inovação do Instituto Senai de Inovação (ISI) Eletrotécnica*
}

\author{
Livia Maria dos Santos ${ }^{\dagger}$ \\ Walter Tadahiro Shima
}

\begin{abstract}
Resumo
A Política Industrial e Tecnológica e de Comércio Exterior (PITCE) de 2003 marca a retomada da política industrial depois de quase 30 anos da sua ausência. A partir dela, outras políticas deram continuidade a busca para alavancar a produção industrial inovativa no país. Entre as diversas estratégias, uma delas foi a construção de uma rede nacional de laboratório para atender os setores industriais em busca de soluções inovativa. Trata-se do Instituto Senai de Inovação (ISI). Este artigo analisa o comportamento dos Institutos de Pesquisas (IPs) a partir das características e ações do ISI Eletroquímica localizado em Curitiba, Paraná. Como metodologia, observou-se primeiramente o comportamento dos IPs com base nos dados do Diretório de Grupos de Pesquisas do CNPq. Na sequência, investigou-se a matriz de decisão do ISI Eletroquímica. Como resultado, nota-se mais do que pesquisa inovativa ou inédita o importante é que a relação IP/empresa seja fundamentalmente rentável para o IP. Em outros termos, o foco na sustentabilidade financeira pode levar os institutos em geral a estar mais orientados pela demanda rentável e muito pouco no desenvolvimento de inovações com graus de ineditismo aplicado.
\end{abstract}

Palavras-chave: Inovação, Política Industrial, Instituto Senai de Inovação (ISI), Relação UniversidadeEmpresa.

JEL: L38, L52, O25

\section{Introdução}

Correntemente os estudos sobre a relação universidade-empresas colocam os institutos de pesquisas (IPs) ao mesmo lado das universidades (Us). Os IPs são vistos como tendo a mesma característica e comportamento das universidades. Como será visto neste

\footnotetext{
*Agradecemos as intituições envolvidas.

†Professora do Instituto Federal do Paraná/Paranaguá. Doutora pelo Programa de Pós-graduação em Políticas Públicas da UFPR. Email: liviams45@gmail.com.

‡Professor do Programa de Pós-graduação em Políticas Públicas da UFPR. Email: waltershima@ufpr.br.
} 
78 A dinâmica de interação institutos de pesquisas/empresas: os condicionantes pró inovação do Instituto Senai de Inovação (ISI) Eletrotécnica

artigo os IPs têm características próprias ligadas à necessidade de prestarem serviços interativos de pesquisas com empresas que não necessariamente são serviços que levam à inovação e sim são serviços focados na venda de uma atividade em busca de retorno financeiro. Este artigo, portanto, busca entender o comportamento dos IPs a partir do estudo das características e ações do Instituto Senai de Inovação (ISI), particularmente o ISI Eletroquímica localizado em Curitiba, Paraná.

O surgimento deste Instituto não é uma iniciativa isolada, mas sim se insere no âmbito de uma política industrial planejada em busca de autonomização das competências tecnológicas instaladas nas firmas do país. As políticas industriais têm um histórico de oscilações no Brasil. Em alguns períodos houve amplo estímulo para o desenvolvimento de inovações em determinadas áreas, como por exemplo, setores bélicos, e posteriormente, houve uma queda nos estímulos, que acompanharam a queda do crescimento econômico somado à inflação crescente, o que ocorreu na década de 1980/90. E por fim, na década de 2000 passa novamente a haver incentivos à inovação, até o início da atual crise econômica.

O ISI é uma rede de laboratórios de Inovação (de diversas áreas do conhecimento) espalhados pelos diversos estados do país criados pelo SENAI como suporte à Empresa Brasileira de Pesquisa e Inovação Industrial (EMBRAPII) criada em 2013 pelo Governo Federal. Em princípio, esses laboratórios têm como objetivo desenvolver pesquisa aplicada e contribuir com as empresas para soluções de problemas de modo inovativo. Desse modo, busca manter no seu corpo de trabalho pesquisadores altamente qualificados, instalações, equipamentos e máquinas específicas a cada área de especialização de cada um dos laboratórios. Cada laboratório espalhado pelo território brasileiro tem uma especialidade. O primeiro inaugurado foi em Curitiba, na área de Eletroquímica e é o que será analisado nesse artigo.

Apesar de existir diversos laboratórios, foi analisado apenas o ISI de Eletroquímica, e a partir dele, analisou-se qual sua contribuição como agente propulsor de inovações. Para realizar a pesquisa, foi feita uma revisão das políticas industriais para inovação até a década de 2013, depois foi analisado os índices de parceria entre institutos de pesquisa e empresas entre 2002 e 2016 no Diretório de Grupos de Pesquisa (DGP), identificando o cenário nacional, e por fim, foram identificadas as estratégias de operação do ISI Eletroquímica que poderiam estimular o avanço de desenvolvimento de técnicas inovativas.

Esse artigo está dividido em 5 partes, a partir da introdução, seguido de um panorama histórico sobre as políticas industriais/de inovação no país para mostrar que o ISI é fruto de um processo evolutivo de política industrial, na sequência, foi realizada uma análise dos dados do DGP e por fim, analisou-se os mecanismos de decisão do ISI e seu impacto na aceleração da inovação no país. Ao final, foi feita a conclusão.

Econômica-Niterói, v. 20, n. 1, p. 77-97. Junho, 2018 


\section{A Evolução das Políticas Industriais de Inovação no Brasil}

O desenvolvimento industrial no Brasil esteve fortemente marcado pela atuação do setor público sendo que desde o primeiro reinado até o período militar houve 5 ondas de ações pró indústria (SUZIGAN; ALBUQUERQUE; CARIO, 2011).

A primeira onda teve início em 1808, quando foram criados cursos de anatomia e cirurgia no Rio de Janeiro e Salvador, o Laboratório Químico Prático do Rio de Janeiro em 1812 e o Museu Real, onde se abrigava o primeiro laboratório de Física e Química do Brasil. No Segundo Reinado, destaca-se a criação de museus de história natural, observatório astronômico, a Comissão Geológica Imperial, Museu Arqueológico e Etnográfico do Pará (1866) e a Escola Superior de Agricultura Luiz de Queiroz (1901), entre outros (SUZIGAN; ALBUQUERQUE; CARIO, 2011; SCHWARTZMAN, 1995).

Durante a segunda onda, entre 1920 e 1934, foi fundada a Universidade de São Paulo (USP) e a Universidade Federal do Rio de Janeiro (UFRJ) (DIAS, 2009). A industrialização na década de 1930 criou a necessidade de modernização do aparelho administrativo, bem como dos instrumentos de ação governamental. Para suprir essa carência foi preciso criar universidades e institutos de pesquisa adequando o sistema de C\&T às necessidades do setor produtivo (SUZIGAN; ALBUQUERQUE; CARIO, 2011).

Na terceira onda, durante o primeiro Governo Vargas, foram construídos alguns organismos estatais voltados à indústria e à atividade extrativa, como o Instituto de Pesquisa Tecnológica (SUZIGAN; ALBUQUERQUE; CARIO, 2011; DIAS, 2009).

A quarta onda pertence ao período pós-guerra. Em 1949 é criado o Centro Brasileiro de Pesquisas Físicas, em 1950, o Instituto Tecnológico da Aeronáutica, e, em seguida, o Centro Tecnológico da Aeronáutica. Em 1951, foi criado o Conselho Nacional de Desenvolvimento Científico e Tecnológico (CNPq) e a Coordenação de Aperfeiçoamento de Pessoal de Nível Superior (Capes). Antes do Golpe Militar, na década de 1960, houve ainda a criação da Universidade de Brasília e da FAPESP (Fundação de Amparo à Pesquisa do Estado de São Paulo). Em 1962 começa a fazer parte do país a elaboração e aplicação de políticas de C\&T (LIMA, 2009). Por fim, a quinta onda se refere ao período do Golpe Militar que envolve a criação de centros de pesquisa nas empresas estatais, como o Centro de Pesquisas e Desenvolvimento Leopoldo Américo Miguez de Mello (CENPES) da Petrobrás, o Centro de Pesquisa e Desenvolvimento em Telecomunicações (CPqD) da Telebrás, e a fundação Embrapa (1973). Foi criado o Fundo de Desenvolvimento Tecnológico (Funtec) em 1964, administrado pelo Banco Nacional do Desenvolvimento Econômico (BNDE), que havia sido criado em 1952. Desse fundo nasceu a Financiadora de Estudos e Projetos (FINEP) em 1965, que teria importante papel na coordenação de ações governamentais na área de financiamento a $C \& T$.

O fim do ciclo expansivo correspondente a esta quinta onda ocorre no início dos anos $80 \mathrm{com}$ as entregas e demarragem dos investimentos oriundos no II PND. Neste momento, a economia brasileira se encontra com esgotamento da capacidade de investimento do 
80 A dinâmica de interação institutos de pesquisas/empresas: os condicionantes pró inovação do Instituto Senai de Inovação (ISI) Eletrotécnica

Estado com elevado déficit fiscal, endividamento externo com déficit na balança de pagamentos correntes e escalada inflacionária o que leva o Governo, de forma errônea, ao exclusivo combate à inflação por meio de políticas ortodoxas recessivas e cortes nos gastos públicos. As demais políticas públicas são gradativamente abandonadas de tal forma que durante todo os anos 80 e 90 há um total retrocesso social, econômico e tecnológico conquistado nas décadas anteriores.

No fim da década de 1990 e início da década de 2000, o Brasil continuava sem uma política industrial efetiva. Depois de quase 30 anos sem política industrial, somente em 2004 passa haver uma ação coordenada entre diversos ministérios, instituições públicas e agências e conselhos industriais para dinamizar a estrutura produtiva do país com o lançamento das diretrizes da Política Industrial e Tecnológica e de Comércio Exterior (PITCE). Esta marcou de modo significativo as ações públicas de fomento à indústria, dando início a um ciclo de diversas ações coordenadas com objetivo de ampliar a capacidade inovativa do país, o que poderia alterar sua trajetória econômica em longo prazo. Na sequência com ampliações, em 2008, é lançada a Política de Desenvolvimento Produtivo (PDP) e em 2011, o Plano Brasil Maior (PBM). Após as ondas, até o período da ditadura militar, houve outros eventos que estimularam o desenvolvimento industrial do país. A tabela 1 sintetiza a linha do tempo da trajetória das políticas industriais.

Tabela 1 - Evolução das Políticas Industriais do Brasil, 2003-2013

\begin{tabular}{lccccccc}
\hline ANO DE LANÇAMENTO & 1974 & 2003 & 2007 & 2008 & 2011 & 2012 & 2013 \\
\hline POLITICA OU AÇÃO & II PND & PITCE & PACTI & PDP & PBM & ENCTI & $\begin{array}{c}\text { EMBRAPII } \\
\text { /ISI }\end{array}$ \\
\hline
\end{tabular}

Fonte: os autores

O PBM tinha um foco na política industrial, tecnológica, de serviços e de comércio exterior durante o período de 2011 a 2014. Dentro da articulação entre essas políticas buscava o estímulo à inovação e à produção nacional para alavancar a competitividade da indústria nos mercados interno e externo por meio da mobilização das forças produtivas e das competências presentes nas empresas, na academia e na sociedade. Mais especificamente, o PBM atuava por meio de financiamentos, aumentos de recursos à inovação, e uma agenda exclusiva para atender as demandas dos setores inovativos (BRASIL, 2011b).

Dentro de suas ações o PBM incluía a Estratégia Nacional de Ciência, Tecnologia e Inovação 2011-2014 (ENCTI), que teve continuidade com a ENCTI 2016-2019, que é o programa do Governo voltado para a tecnologia. A ENCTI se apoiou na experiência de planejamento dos PBDCTs dos anos 70 (citados anteriormente), da criação do MCT\&I, juntamente com o estabelecimento das Conferências Nacionais de Ciência e Tecnologia (CNCT) e o advento dos Fundos Setoriais, no fim dos anos 90 (BRASIL, 2011a).

Econômica-Niterói, v. 20, n. 1, p. 77-97. Junho, 2018 


\subsection{A Articulação Público e Privado a partir da Criação da EMBRAPII}

Outro elemento importante da política industrial foi o lançamento da EMBRAPII, qualificada como uma Organização Social pelo Poder Público Federal desde setembro de 2013. A assinatura do Contrato de Gestão com o MCTI ocorreu em 2 de dezembro de 2013, tendo o MEC como instituição interveniente. Os dois órgãos federais repartem igualmente a responsabilidade pelo seu financiamento (EMBRAPII, 2015). Ela foi criada a partir das propostas da Mobilização Empresarial pela Inovação (MEI), movimento liderado pela Confederação Nacional das Indústrias (CNI) visando contribuir para uma maior articulação institucional entre os setores público e privado, entre universidades, centros de pesquisa e empresas no desenvolvimento de tecnologias inovadoras, de modo a complementar a atuação das agências de fomento existentes e as ações em curso.

Seu objetivo principal era a ampliação da articulação entre universidades, centros de pesquisa e empresas no desenvolvimento de tecnologias inovadoras, com ênfase na fase final do desenvolvimento de produtos, em atividades como escalonamento, prova de conceito e planta demonstração. A EMBRAPII teve como característica principal o balizamento de seus programas pelo atendimento às demandas dos setores associados, não se constituindo de laboratórios próprios, fazendo uso intensivo das redes de institutos e centros de pesquisa já existentes, com capilaridade e competência comprovada em projetos com empresas (BRASIL, 2011a).

Sendo assim, a EMBRAPII constituiu-se como um modelo de aproximação de laboratórios e universidades públicas que visam se aproximar das empresas para desenvolvimento de pesquisa aplicada. Como suporte à EMBRAPII o Serviço Nacional da Indústria (SENAI) vem criando uma rede de laboratórios de Inovação espalhados pelos diversos estados do país que são os Institutos SENAI de Inovação (ISI).

A forma de organização desses institutos foi inspirada no Instituto Fraunhofer da Alemanha, que consiste em um sistema de organizações de inovação tecnológica que têm apoio do governo e são direcionadas à pesquisa aplicada, atendendo as demandas das indústrias, e são referência na criação de soluções inovadoras.

A expectativa é que até 2018 estarão prontos 25 ISIs no país, em 12 estados, sendo que até o momento, 21 já estão em funcionamento, com 150 projetos contratados, e suas construções já receberam mais de R \$ 6 bilhões em investimento até 2015 (SENAI, 2015). Os institutos contam com pesquisadores mestres e doutores e uma robusta infraestrutura, destinados a atender demandas do setor industrial no desenvolvimento de tecnologias e criando soluções. A tabela 2 abaixo, pode-se observar a distribuição dos ISIs no país e suas relativas áreas de atuação.

Considerando que o ISI é um instituto de pesquisa (IP) e busca interação como setor produtivo, conforme apresentado anteriormente, a seguir faz-se uma análise do comportamento geral dos IPs no Brasil e seu relacionamento com empresas para logo analisar o caso das características do processo interativo especifico do ISI Eletrotécnica, (Paraná). 
82 A dinâmica de interação institutos de pesquisas/empresas: os condicionantes pró inovação do Instituto Senai de Inovação (ISI) Eletrotécnica

Tabela 2 - Distribuição das unidades do ISI pelos estados do brasil

\begin{tabular}{|c|c|c|}
\hline SUL (7 unidades) & $\begin{array}{l}\mathrm{RS} \\
\mathrm{SC} \\
\mathrm{PR}\end{array}$ & $\begin{array}{l}\text { Eng. De Polímeros } \\
\text { Metal-mecânica } \\
\text { Sistemas de Manufaturas } \\
\text { Laser } \\
\text { Sistemas Embarcados } \\
\text { Eletroquímica } \\
\text { Eng. De Estruturas }\end{array}$ \\
\hline SUDESTE (10 unidades) & $\begin{array}{l}\text { SP } \\
\text { RJ } \\
\text { MG }\end{array}$ & $\begin{array}{l}\text { Micro manufatura } \\
\text { Materiais Avançados } \\
\text { Biotecnologia } \\
\text { Sistemas Virtuais } \\
\text { Química Verde } \\
\text { Biossintéticos } \\
\text { Eng. De Superfícies } \\
\text { Metalurgia } \\
\text { Processamento Mineral } \\
\text { Sistemas Elétricos }\end{array}$ \\
\hline CENTRO OESTE ( 1 unidade) & MS & Biomassa \\
\hline NORDESTE (5 unidades) & $\begin{array}{l}\mathrm{BA} \\
\mathrm{PE} \\
\mathrm{RN}\end{array}$ & $\begin{array}{l}\text { Automação } \\
\text { Conformação e União } \\
\text { Logística } \\
\text { Tecnologia da Informação } \\
\text { Energias Renováveis }\end{array}$ \\
\hline NORTE (2 unidades) & $\begin{array}{l}\mathrm{PA} \\
\mathrm{AM}\end{array}$ & $\begin{array}{l}\text { Tecnologias Minerais } \\
\text { Microeletrônica }\end{array}$ \\
\hline
\end{tabular}

Fonte: os autores 


\section{O Processo Interativo dos Institutos de Pesquisa (IP) com o Setor Produtivo no Brasil: Uma Análise dos Dados do Diretório dos Gru- pos de Pesquisa do CNPq}

Essa sessão consiste em analisar os avanços da interação entre universidades (Us)/instituto de pesquisa (IPs) com empresas. Os dados foram analisados no período entre 2002 a 2016, no Brasil, e foi dado maior enfoque às interações entre IPs e empresas.

Em termos evolutivos, de acordo com a a tabela 3 o número de GPs com relacionamento, tanto de Us como de IPs cresceu mais do que o total de GPs da federação e os GPs das Us com relacionamento cresceram bem mais do que dos IPs $(18,46 \%$ contra $10,57 \%)$ o que significa que as Us passaram a ter mais relacionamentos que os IPs, aumentando sua relevância na contribuição produtiva. Ao mesmo tempo, os GPs total da federação dos IPs cresceram apenas 0,69\% a.a., e os GPs das Us total da federação cresceram 7,68\%. Ou seja, os IPs perderam relevância relativa ao longo do tempo. Entretanto, em termos do período, os IPs se relacionam mais relativamente às Us. Em 2002, os GPs com relacionamento dos IPs eram 14,70\% (a/b) e das Us eram 8,75\% (a/b) do total e em 2016 era $54,46 \%$ (c/d) dos IPs contra 33,29\% (c/d) das Us. Ou seja, comparativamente os GPs com relacionamento das Us cresceram mais do que dos IPs, mas em termos relativos os IPs se relacionam mais. Para que a relação a/b das U fosse maior do que dos IPs a TCAC das Us $(18,46 \%)$ teria que ser muito maior. Ou os GPs com relacionamento das Us teriam que ter crescido a uma taxa muito maior.

Para uma análise mais especifica, relacionando grandes áreas, GP, L, P, regiões, relacionamento com empresas, etc. os dados são obtidos a partir do Plano Tabular (CNPQ, 2017) que como já é amplamente sabido foi descontinuado a partir de 2010. Portanto, só possível fazer cruzamentos de dados do Diretórios de Grupos de Pesquisas no Brasil até 2010. No total do país a tabela 4 mostra, para 2010, as quantidades de GP, L e P para as grandes áreas. Obviamente as quantidades dos IP são absolutamente inferiores aos das U. Entretanto, as relações L/GP e P/GP são superiores nos IPs, o que significa que os IPs tendem a ter linhas mais especificas ou focos mais específicos (uma vez que as linhas e os pesquisadores se concentram em menos quantidades de GPs). Para os IPs nota-se a nítida concentração de atividades nas Ciências Agrárias e Ciências Biológicas. Por outro lado, nas Us não há essa nítida concentração de atividades. Há grande dispersão dos números em todas as variáveis, com um relativo destaque para Ciências Humanas, Engenharias e Ciências da Saúde, o que difere totalmente dos IPs. Sendo assim, é óbvio que os valores das relações L/GP, P/GP e P/L sejam inferiores aos dos IPs. Isso reflete claramente o sentido de liberdade e abertura das universidades nos temas de pesquisas, enquanto que os IPs podem estar focados em grandes áreas cujas pesquisas sejam mais promissoras em termos de rentabilidade, auto sustentação financeira, resposta imediata a pesquisas sob demanda, soluções de curto prazo, etc.

A tabela 5 apresenta os mesmos dados da tabela anterior para o Paraná. Da mesma 
84 A dinâmica de interação institutos de pesquisas/empresas: os condicionantes pró inovação do Instituto Senai de Inovação (ISI) Eletrotécnica

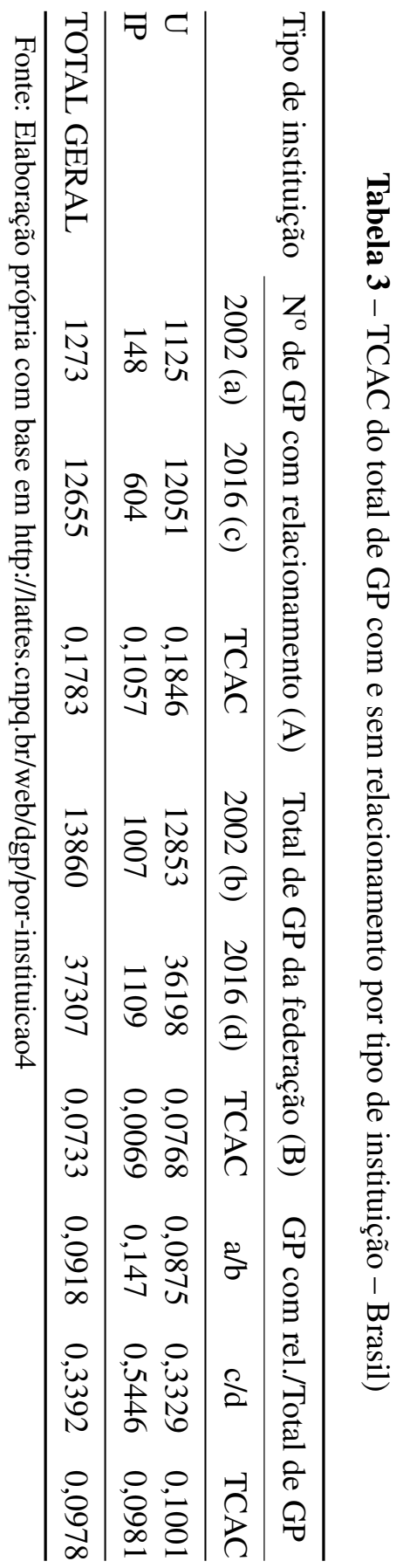

Econômica-Niterói, v. 20, n. 1, p. 77-97. Junho, 2018 
Tabela 4 - Total de GP, linhas de pesquisa $(\mathrm{L})$ e pesquisadores $(\mathrm{P})$ e relação L/GP, P/GP, P/L - Brasil - 2010)

\begin{tabular}{lcccccc}
\hline Grande área & $\mathrm{GP}$ & $\mathrm{L}$ & $\mathrm{P}$ & $\mathrm{L} / \mathrm{GP}$ & $\mathrm{P} / \mathrm{GP}$ & $\mathrm{P} / \mathrm{L}$ \\
\hline Ciências Agrárias & 49 & 330 & 552 & 6,73 & 11,27 & 1,67 \\
Ciências Biológicas & 15 & 93 & 145 & 6,2 & 9,67 & 1,56 \\
Ciências Exatas e da Terra & 8 & 39 & 61 & 4,88 & 7,63 & 1,56 \\
Ciências Humanas & 1 & 1 & 3 & 1 & 3 & 3 \\
Ciências Sociais Aplicadas & 3 & 14 & 20 & 4,67 & 6,67 & 1,43 \\
Engenharias & 17 & 60 & 121 & 3,53 & 7,12 & 2,02 \\
TOTAL IP & 93 & 537 & 902 & 5,77 & 9,7 & 1,68 \\
\hline Ciências Agrárias & 259 & 992 & 2146 & 3,83 & 8,29 & 2,16 \\
Ciências Biológicas & 225 & 926 & 1666 & 4,12 & 7,4 & 1,8 \\
Ciências da Saúde & 325 & 1072 & 2321 & 3,3 & 7,14 & 2,17 \\
Ciências Exatas e da Terra & 245 & 966 & 1499 & 3,94 & 6,12 & 1,55 \\
Ciências Humanas & 414 & 1121 & 3033 & 2,71 & 7,33 & 2,71 \\
Ciências Sociais Aplicadas & 289 & 722 & 1930 & 2,5 & 6,68 & 2,67 \\
Engenharias & 290 & 1233 & 1920 & 4,25 & 6,62 & 1,56 \\
Linguística, Letras e Artes & 124 & 297 & 804 & 2,4 & 6,48 & 2,71 \\
TOTAL U & 2171 & 7329 & 15319 & 3,38 & 7,06 & 2,09 \\
\hline TOTAL GERAL & 2264 & 7866 & 16221 & 3,47 & 7,16 & 2,06 \\
\hline
\end{tabular}

Fonte: elaboração própria com base em

http://dgp.cnpq.br/planotabular/index.jsp

forma que no Brasil, o Paraná segue a mesma tendência de comportamento em que os IPs concentram claramente atividades em Ciências Agrárias e Ciências Biológicas e nas Us há bastante dispersão dos números entre as grandes áreas, mas com certo destaque para as áreas de Ciências Humanas, Ciências Exatas e da Terra e Ciências da Saúde, diferente do Brasil. Os valores das relações L/G, P/G e P/L nos IP são superiores aos valores das Us pelas, supostamente, mesmas razoes e fundamentos das Universidades.

A tabela 6 mostra que há relativa diferença no comportamento entre os IPs e as Us. Conforme já apontado anteriormente na tabela 3, os IPs se relacionam relativamente mais que as Us. Aqui isso também fica claro quando se verifica a densidade que para os IP = 2,63 , superior às $U=1,99$. Para os IPs os dois tipos de relacionamento mais importantes são o Rel2 e Rel7, o que indica também algo já apontado anteriormente que os IPs são solution providers, uma vez que, precisam buscar sua sobrevivência baseada em rentabilidade, portanto, esses dois tipos indicam exatamente o caráter de se apresentar soluções imediatas dentro de uma lógica de entrega do produto contra o pagamento imediato. As duas áreas com mais tipos de relacionamento são Ciências Agrárias e Engenharias dentro desses mesmo dois tipos de relacionamento (Rel2 e Rel7). Por outro lado, as Us com uma perspectiva de relativa maior liberdade têm densidade menor $(1,99)$, considerando 
86 A dinâmica de interação institutos de pesquisas/empresas: os condicionantes pró inovação do Instituto Senai de Inovação (ISI) Eletrotécnica

Tabela 5 - Total de GP, linhas de pesquisa (L) e pesquisadores (P) e relação L/GP, P/GP, P/L - Paraná - 2010)

\begin{tabular}{lcccccc}
\hline Grande área & $\mathrm{G}$ & $\mathrm{L}$ & $\mathrm{P}$ & $\mathrm{L} / \mathrm{G}$ & $\mathrm{P} / \mathrm{G}$ & $\mathrm{P} / \mathrm{L}$ \\
\hline Ciências Agrárias & 58 & 337 & 460 & 5,81 & 7,93 & 1,36 \\
Ciências Biológicas & 32 & 132 & 225 & 4,13 & 7,03 & 1,7 \\
Ciências da Saúde & 6 & 17 & 22 & 2,83 & 3,67 & 1,29 \\
Ciências Exatas e da Terra & 24 & 59 & 134 & 2,46 & 5,58 & 2,27 \\
Ciências Humanas & 4 & 8 & 19 & 2 & 4,75 & 2,38 \\
Ciências Sociais Aplicadas & 10 & 18 & 44 & 1,8 & 4,4 & 2,44 \\
Engenharias & 25 & 68 & 134 & 2,72 & 5,36 & 1,97 \\
Tecnologias & 6 & 15 & 29 & 2,5 & 4,83 & 1,93 \\
TOTAL IP & 165 & 654 & 1067 & 3,96 & 6,47 & 1,63 \\
\hline Ciências Agrárias & 370 & 1135 & 1963 & 3,07 & 5,31 & 1,73 \\
Ciências Biológicas & 389 & 1121 & 1903 & 2,88 & 4,89 & 1,7 \\
Ciências da Saúde & 419 & 1158 & 2248 & 2,76 & 5,37 & 1,94 \\
Ciências Exatas e da Terra & 457 & 1445 & 2126 & 3,16 & 4,65 & 1,47 \\
Ciências Humanas & 543 & 1279 & 3197 & 2,36 & 5,89 & 2,5 \\
Ciências Sociais Aplicadas & 394 & 852 & 2067 & 2,16 & 5,25 & 2,43 \\
Engenharias & 310 & 1038 & 1488 & 3,35 & 4,8 & 1,43 \\
Linguística, Letras e Artes & 156 & 340 & 827 & 2,18 & 5,3 & 2,43 \\
Não informado & 3 & 3 & 7 & 1 & 2,33 & 2,33 \\
Tecnologias & 21 & 31 & 84 & 1,48 & 4 & 2,71 \\
TOTAL U & 3062 & 8402 & 15910 & 2,74 & 5,2 & 1,89 \\
\hline TOTAL GERAL & 3227 & 9056 & 16977 & 2,81 & 5,26 & 1,87 \\
\hline
\end{tabular}

Fonte: elaboração própria com base em

http://dgp.cnpq.br/planotabular/index.jsp

que também devem realizar ensino e extensão (ou seja, não se dedicam exclusivamente à pesquisa) e as áreas de maior densidade também são Engenharias e Ciências Agrárias (com ordem inversa dos IP). Os três tipos de relacionamento predominantes são Rel2, Rel1 e Rel7, também dentro de Engenharias e Ciências Agrárias. Aqui também existe a lógica de soluções imediatas, mas com um certo apontamento para pesquisas de maior folego quando o segundo maior tipo de relacionamento é o Rel1. Porém, no gráfico 1, que mostra a distribuição do total de relacionamento por tipo de relacionamento, se verifica que em geral tanto Us quanto IPs têm o mesmo tipo de relacionamento: primeiro Rel2, Rel7, Rel1, Rel9 e Rel10, sendo que os IPs apresentam mais relacionamentos.

No Paraná o comportamento dos grupos é relativamente o mesmo do Brasil. Conforme a 6 a densidade dos IPs também é maior que das Us. A quantidade de Grandes Áreas dos IPs relatadas como tendo relação são menores do que no Brasil, enquanto as Us continuam tendo relação em todas as Grandes Áreas. Os tipos de relacionamento pre- 

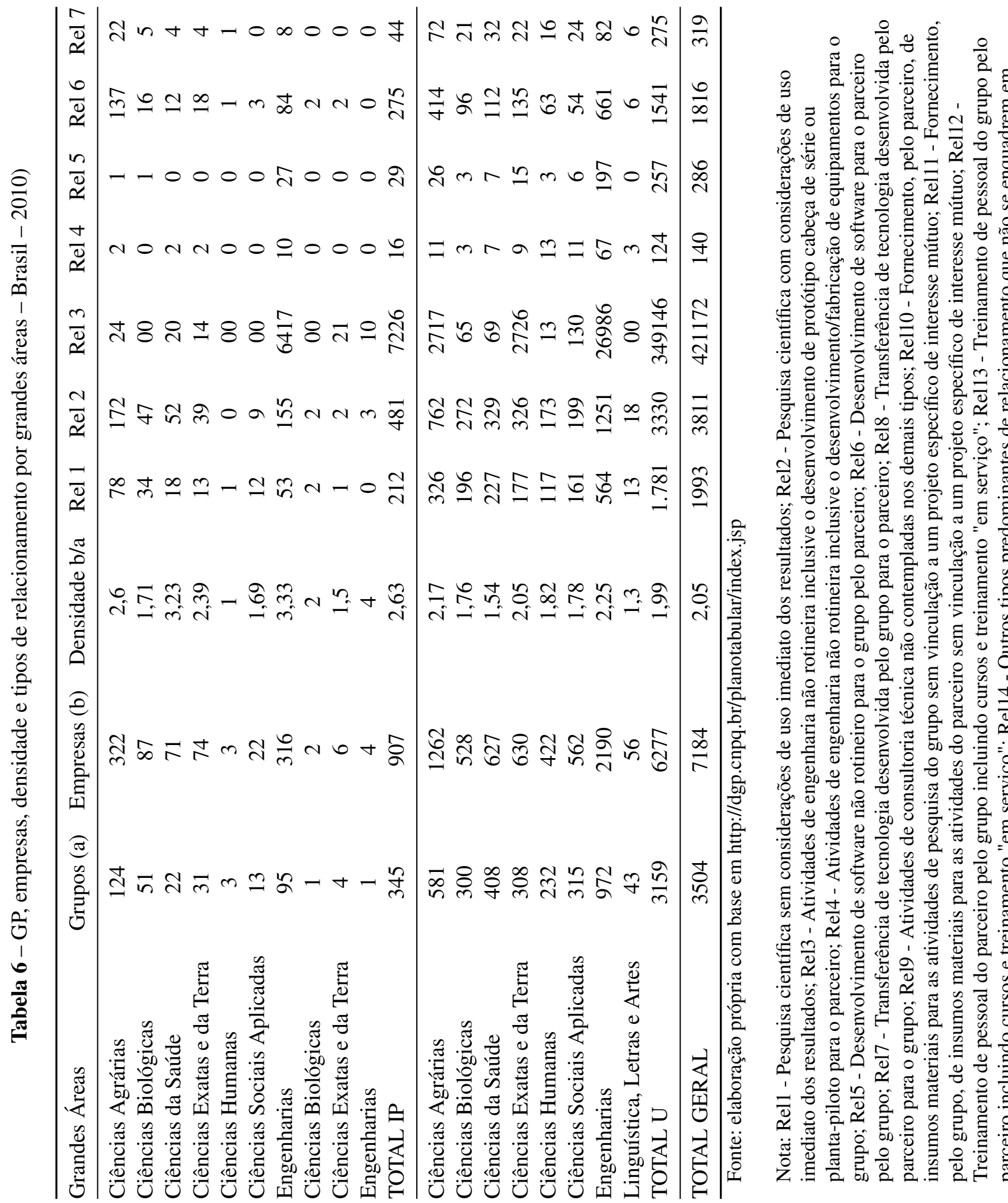

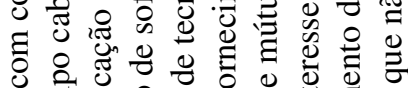

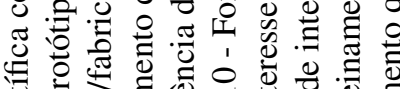

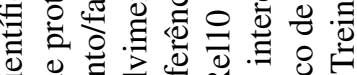

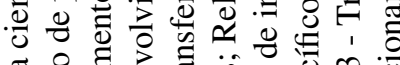

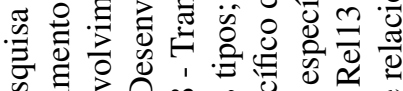

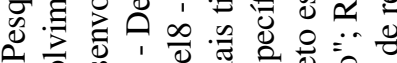

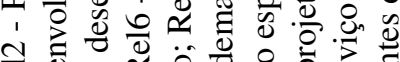

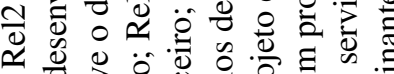

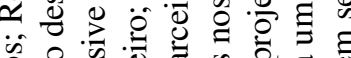

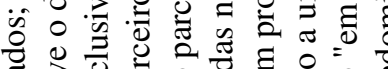

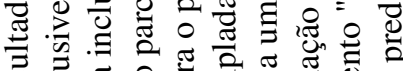
ڤั) क.

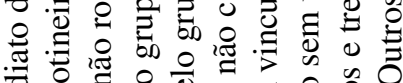
ఫั

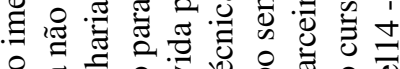

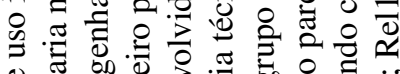
ช 正 记

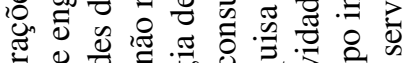

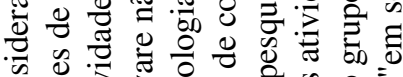

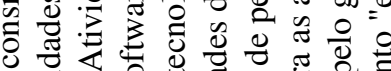

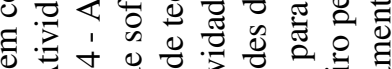

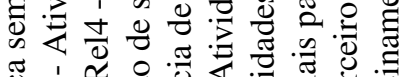

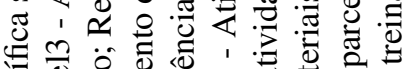

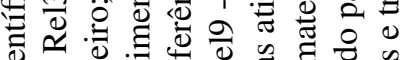
पis .

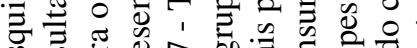

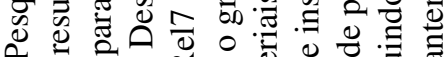

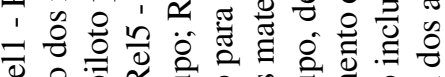
ॠ

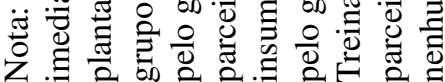


88 A dinâmica de interação institutos de pesquisas/empresas: os condicionantes pró inovação do Instituto Senai de Inovação (ISI) Eletrotécnica

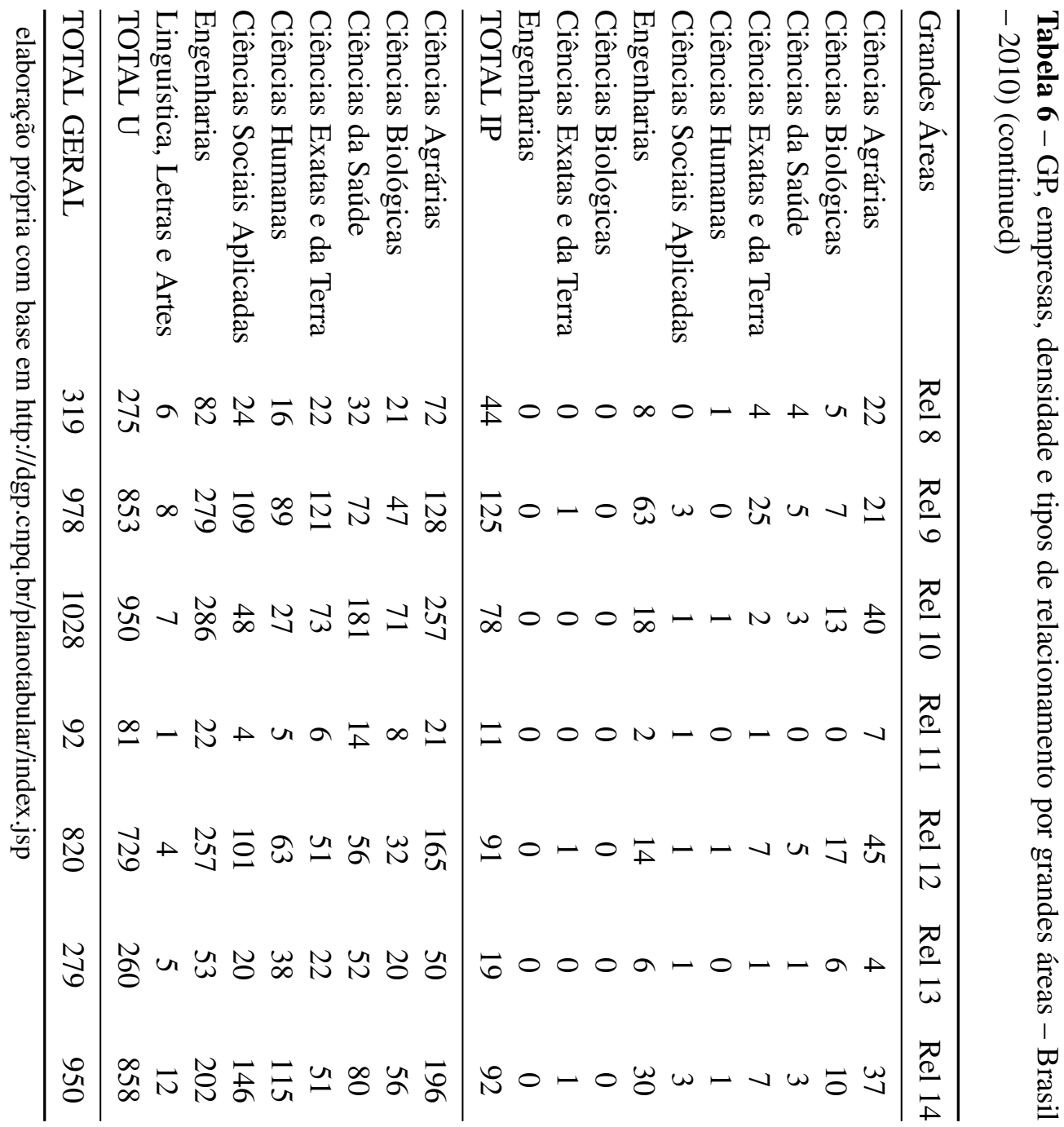

Econômica-Niterói, v. 20, n. 1, p. 77-97. Junho, 2018 
Figura 1 - Índice da distribuição do número de relacionamentos por tipo de relacionamento Brasil - 2010

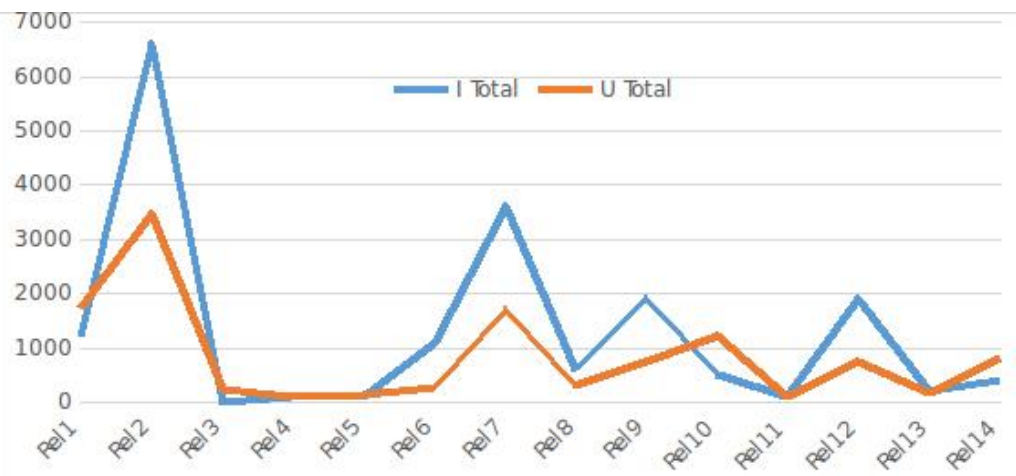

Fonte: elaboração própria com base na tabela 3

dominantes em ordem de relevância, de acordo com o gráfico 2 são: Rel2, Rel7, Re19, Rel1 e Rel12. No Paraná ocorre uma variação de tipo de relacionamento maior do que no Brasil.

Figura 2 - Índice da distribuição do número de relacionamentos por tipo de relacionamento Paraná - 2010

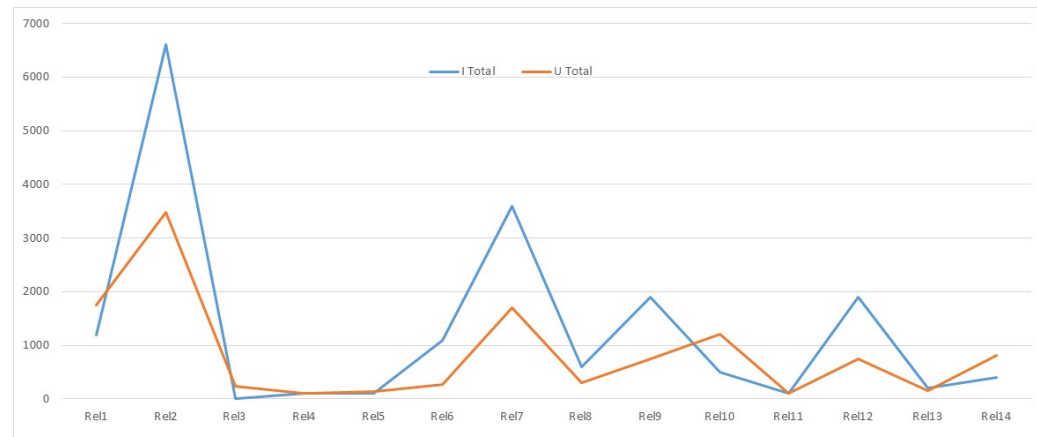

Fonte: elaboração própria com base na tabela4

Em síntese:

- Obviamente a quantidade total de IPs e IPs com relacionamento são absolutamente inferiores à quantidade de Us. Porém em termos relativos, os IPs se relacionam mais do que as Us. A densidade de relacionamento dos IPs é maior do que das Us.

- As linhas e pesquisadores dos IP são mais concentrados em focos mais específicos nas áreas de Ciências Agrárias e Ciências Biológicas. Nos dados do Paraná se 
90 A dinâmica de interação institutos de pesquisas/empresas: os condicionantes pró inovação do Instituto Senai de Inovação (ISI) Eletrotécnica

observa a mesma situação.

- Os IP têm mais relacionamentos do tipo Rel2 - Pesquisa científica com considerações de uso imediato dos resultados e Rel7 - Transferência de tecnologia desenvolvida pelo grupo para o parceiro que indica que atuam mais na perspectiva de soluções imediatas por conta da necessidade se obter rentabilidade. No Paraná o comportamento dos IPs é bastante similar.

Sendo assim, a próxima sessão analisa o comportamento do ISI Eletroquímica a luz dos elementos verificados nos dados anteriores focando na perspectiva de interação para a inovação.

\section{Os elementos de avaliação de Projeto do ISI Eletrotécnica como Me- canismo Reativo à Inovação}

O objetivo dessa sessão é analisar o comportamento dos ISIs como instrumento de alavancagem da inovação. Para responder a esse objetivo, foi feito um estudo do ISI de Eletroquímica sobre suas estratégias de decisão e seu alinhamento com a proposta das políticas industriais nacionais.

Uma vez que se trata de um IP que trabalha exclusivamente com interação por meio de demandas é necessário que cada demanda seja previamente analisada em termos de viabilidade e dos setores industriais prioritários. A análise baseia-se em uma matriz de decisão, e seus itens e pesos seguem na tabela 7 em que a viabilidade econômico-financeira tem peso 65 e a Tecnológica, 30, demonstrando o fato de que os IP, mais do que pesquisa per se precisam realizar negócios que tenham foco em atividades interativas que garantam a sobrevivência da instituição. Ao mesmo tempo que só pode realizar aquilo cujas competências técnicas já estejam instaladas ou sejam de relativo fácil acesso interagindo com universidades nacionais ou internacionais, ter rotas tecnológicas dominadas, etc.

Na medida em que o aspecto econômico-financeiro tem um peso muito alto há sempre um risco de que eventuais projetos de inovação com um caráter mais radical ou de alto impacto em termos aumento significativo de conhecimento ou desenvolvimento de novas rotas tecnológicas sejam inviabilizadas em função de eventual baixa atratividade econômico-financeira. Ou seja, eventuais projetos que não tenham um apelo de sustentabilidade financeira inicial, mas com alto impacto tecnológico correm o risco de serem abandonados por não terem fonte de financiamento viável. Portanto, um projeto que atenda completamente a todos os itens da categoria Tecnológica e apenas parcialmente a econômico-financeira pode ser preterido a um projeto em situação inversa (atenda completamente o setor econômico-financeiro e parcialmente o tecnológico que eventualmente poderia ser mais inovador na medida em que poderia implicar alto grau de complexidade, por exemplo).

Econômica-Niterói, v. 20, n. 1, p. 77-97. Junho, 2018 
Tabela 7 - Matriz de Decisão do ISI Eletroquímica

\begin{tabular}{|c|c|c|c|c|}
\hline Categoria & $\mathrm{N}^{\mathrm{o}}$. & Itens de avaliação & Pesos & Prioridade \\
\hline \multirow[t]{11}{*}{ Tecnológica } & 1 & $\begin{array}{l}\text { Grau de complexidade de } \\
\text { execução }\end{array}$ & 10 & $1=$ alto \\
\hline & & & & $5=$ baixo \\
\hline & 2 & Equipe ISI domina assunto & 5 & $\begin{array}{l}1=\text { não } \\
5=\operatorname{sim}\end{array}$ \\
\hline & 3 & $\begin{array}{l}\text { Parcerias com universidades } \\
\text { nacionais }\end{array}$ & 3 & $1=$ não \\
\hline & & & & $5=\operatorname{sim}$ \\
\hline & 4 & Parcerias com outros ISIs & 3 & $1=$ não \\
\hline & & & & $5=\operatorname{sim}$ \\
\hline & 5 & $\begin{array}{l}\text { Parcerias universidades in- } \\
\text { ternacionais }\end{array}$ & 3 & 1 = não \\
\hline & & & & $5=\operatorname{sim}$ \\
\hline & 6 & $\begin{array}{l}\text { Compartilhamento de rota } \\
\text { tecnológica }\end{array}$ & 6 & 1 = não \\
\hline & & & & $5=\operatorname{sim}$ \\
\hline PESO DA CATEGORIA & & & 30 & XXX \\
\hline \multirow[t]{14}{*}{ Econômica- financeira } & 7 & Negociação & 5 & 1 = discussão inicial \\
\hline & & & & $\begin{array}{l}3=\text { discussão técnica } \\
5=\text { aceitou proposta }\end{array}$ \\
\hline & 8 & $\begin{array}{l}\text { Sustentabilidade financeira } \\
\text { ISI }\end{array}$ & 50 & $1=$ até $3 \%$ \\
\hline & & & & $2>3 \%<6 \%$ \\
\hline & & & & $3=6 \%<9 \%$ \\
\hline & & & & $\begin{array}{l}4=9 \%<11 \% \\
5-11 \%<15 \%\end{array}$ \\
\hline & 9 & $\begin{array}{l}\text { Contrapartida financeira in- } \\
\text { fraestrutura }\end{array}$ & 5 & $\begin{array}{l}5=11 \%<15 \% \\
1=\text { baixo }\end{array}$ \\
\hline & & & & $5=$ alto \\
\hline & 10 & Demanda da indústria & 1 & $\begin{array}{l}1=\text { baixo } \\
5=\text { alto }\end{array}$ \\
\hline & 11 & Royalties & 5 & $1=0 \%$ \\
\hline & & & & $22 \%$ \\
\hline & & & & $3>2 \%<3 \%$ \\
\hline & & & & $\begin{array}{l}4=3 \%<5 \% \\
5=5 \%\end{array}$ \\
\hline & & & & $\begin{array}{l}55 \% \\
\mathrm{XYX}\end{array}$ \\
\hline PESO DA CATEGORIA & & & 65 & XXX \\
\hline
\end{tabular}

Fonte: ISI Eletroquímica, 2015 
92 A dinâmica de interação institutos de pesquisas/empresas: os condicionantes pró inovação do Instituto Senai de Inovação (ISI) Eletrotécnica

Tabela 7 - Matriz de Decisão do ISI Eletroquímica (continued)

\begin{tabular}{|c|c|c|c|c|}
\hline Categoria & $\mathrm{N}^{\mathrm{o}}$. & Itens de avaliação & Pesos & Prioridade \\
\hline Estratégica & 12 & $\begin{array}{l}\text { Impacto no mercado ao lançar o pro- } \\
\text { duto }\end{array}$ & 1 & $1=$ baixo \\
\hline & 13 & $\begin{array}{l}\text { Novidade do produto no mercado naci- } \\
\text { onal }\end{array}$ & 1 & $\begin{array}{l}5=\text { alto } \\
1=\text { baixo }\end{array}$ \\
\hline PESO DA CATEGORIA & & & 2 & $\begin{array}{l}5=\text { alto } \\
\mathrm{XXX}\end{array}$ \\
\hline Física & 14 & Infraestrutura e equipamentos do ISI & 1 & $\begin{array}{l}1=\text { baixo } \\
5=\text { alto }\end{array}$ \\
\hline Humana & 15 & $\mathrm{~N}^{\mathrm{o}}$ de pessoas para execução & 1 & $\begin{array}{l}5=\text { compl } . \\
3=\text { parcial } \\
1=\text { incompl. }\end{array}$ \\
\hline
\end{tabular}

Fonte: ISI Eletroquímica, 2015

O gráfico 3 mostra uma situação hipotética do que seria a melhor pontuação que um projeto poderia obter, bem como o valor do peso de cada item de avaliação. $\mathrm{O}$ item sustentabilidade foi retirado, pois seu elevado peso, conforme já discutido, tem capacidade de decidir sozinho por um projeto. Tendo peso 50 a sustentabilidade cria deformidades no gráfico, impedindo a análise de qualquer outro item de decisão.

Além dele, o item complexidade também foi substituído por baixa complexidade, pois é como o ISI considera mais vantajoso esse item. Isso significa que ao avaliar dois projetos com idênticos pesos em todos os outros itens, mas com diferença na complexidade, será preferível o menos complexo porque demanda menos tempo de trabalho, maior facilidade em encontrar a qualificação necessária e menor quantidade de mão de obra. Portanto, um projeto ideal é um de baixa complexidade, o que coloca o ISI como uma instituição não inovadora por princípio.

No gráfico observa-se bem que ser novidade no mercado nacional é pouco relevante, bem como o impacto ao lançar o produto no mercado o que significa baixa relevância de se ter a inovação como foco importante na adoção de projetos. Outro item que apresenta pouca representatividade na tomada de decisão do ISI é a demanda da indústria que também indica pouca relevância da inovação uma vez que, o ISI atua sob demanda e não propondo mudança aos setores industriais. Trata-se de uma atitude reativa e não pró inovação. Outras características que também tiveram pouco peso foram a infraestrutura e a quantidade de pessoas suficiente para a execução do projeto. Esse último item cai em contradição com o mais importante, que é a sustentabilidade (que significa a capacidade de pagar o pessoal envolvido na pesquisa). Ou seja, manter pesquisadores estáveis, indica uma capacitação permanente pró inovação na medida em que conhecimentos se acumu- 
lam ao longo dos projetos, o que implica escala em inovação. Porém, considerando que o ISI depende, em grande parte, de bolsistas de pesquisa adquiridos a partir de editais públicos, logo após a finalização do projeto, grande parte do conhecimento tácito adquirido se perde na saída dos bolsistas. Por outro lado, o item compartilhamento da rota tecnológica assume bastante relevância, em função de que os projetos que implicam cooperação devem resultar em transferência de conhecimento ao ISI.

Figura 3 - SIMULAÇÃO DE UM PROJETO IDEAL PARA O ISI

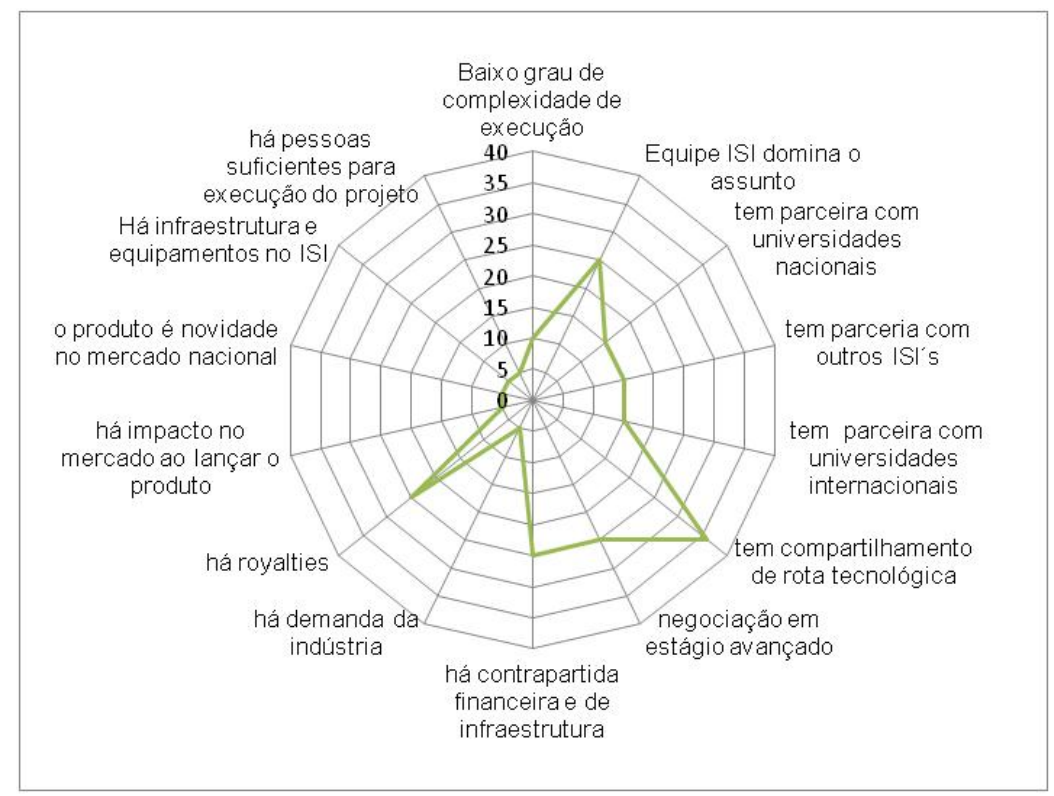

Fonte: ADAPTADO DO ISI, 2015

Outra avaliação importante se refere ao alinhamento das propostas oficiais do ISI com a prática estratégica. A comparação foi feita por meio das informações disponíveis pelo Senai quanto aos seus objetivos e os valores dados a cada um dos itens das diretrizes internas, conforme o gráfico 3. Foi feita uma simulação em que se atribui um valor que oferece o melhor resultado para o ISI. A partir desses resultados, conseguiu-se dimensionar o valor percentual de importância que cada item oferece.

Comparando as premissas públicas do ISI com as diretrizes internas, percebe-se que há alguns desalinhamentos. Enquanto se advoga no site institucional que se promove uma política para tecnologias complexas "serviços tecnológicos de alta complexidade e alto valor agregado" (SENAI, 2015), dentro da diretriz interna percebe-se que são evitadas as tecnologias complexas ou que o grupo não detenha conhecimento. Conforme o gráfico 3 o projeto eleito é de preferência, o com baixo grau de complexidade de execução.

Outro item que está em desacordo com a política institucional oficial é o item que reforça o entusiasmo com "pesquisa aplicada e projetos de inovação tecnológica". Da 
94 A dinâmica de interação institutos de pesquisas/empresas: os condicionantes pró inovação do Instituto Senai de Inovação (ISI) Eletrotécnica

mesma forma, que acima esse item, de acordo com o gráfico 33 é um dos que possui menor valor de decisão. Na mesma categoria, os itens "há impacto no mercado ao lançar o produto" e "o produto é novidade no mercado nacional", juntos não correspondem a $3 \%$ do valor total.

Quanto à "conexão com os principais atores do Sistema Nacional de Inovação" conforme publicado, porém, sua importância é de aproximadamente 10\%. Já o item transferência tecnológica, aumento de performance, redução de riscos tecnológicos, podem ser comparados com o item "compartilhamento da rota tecnológica". Trata-se de um item ao qual é dada muita importância, alinhando-se com a diretriz oficial.

Segundo outra diretriz do ISI "o atendimento dos institutos abrange todo o território nacional, focando no suporte para inovação das empresas de base tecnológica" (SENAI, 2015). Contudo, de acordo com o GRÁFICO 3 a diretriz interna, "há demanda da indústria", assume relevância de menos de $1 \%$, o que ressalta a contradição entre o que é divulgado para o mercado e a efetiva realidade deste IP, fundamentalmente focado em sustentabilidade financeira. Desse modo, por exemplo, se houver entre 10 projetos, 9 não tiverem auto sustentabilidade financeira e apenas um tiver, esse será priorizado.

Esses são aspectos relativos levantados exclusivamente no ISI Eletroquímica, o que não significa necessariamente que ele se replique para a rede do ISI nacional. Nessa lógica do ISI Eletroquímica o importante é a necessidade de manter a estrutura funcionando, consolidar a estrutura como a um todo, tais como marca e confiança das empresas, para posteriormente, numa condição financeira sustentável e boa penetração no mercado (alto volume de atendimento aos setores industriais) conseguir pensar em projetos de inovação propriamente (com graus maiores ou menores de ineditismo). Dentro dessa estratégia de atuação, conforme o gráfico 3, a prioridade é dada para projetos de baixa complexidade e é importante que os novos projetos possam reutilizar os conhecimentos gerados anteriormente, de tal forma, que haja economia de escala nas rotas tecnológicas usadas ou descobertas pelo ISI. Ou seja, os conhecimentos gerados devem ser aproveitados no máximo de projetos possíveis, uma vez que, demanda-se pouca mão de obra, e mesmo assim, pode se ter elevado valor comercial. Isso não significa que nenhum dos dados aponte no sentido de que o ISI ficará estagnado nas tecnologias que já domina, mas sim que ele venderá essa tecnologia para o maior número de compradores. Ainda dentro dessa perspectiva, portanto, a busca pela aproximação a universidades é apenas uma estratégia de apoio secundária, na medida da necessidade de se encontrar competências indisponíveis no Instituto. Não se tem, neste momento, a possibilidade e a perspectiva de se ter uma relação U/IP-empresas como elemento determinante do processo inovativo. É uma possibilidade, mas apenas se for necessária.

Em síntese, mais que buscar inovação, ou promover a relação universidade-empresa, ou atender à indústria, o ISI busca atender seus princípios, que é priorizar o retorno financeiro. Essa situação pode ser padrão para todos os ISIs do país, embora a análise centrou-se em apenas uma unidade. A missão de sua criação é sanar a deficiência inova- 
tiva da indústria, mas tem seu outro papel, de ser sustentável, sendo a missão suprimida pela necessidade de rentabilidade. Ademais do aspecto financeiro e como consequência dele a atuação se concentra em aceitar projetos de baixa relevância inovativa, que usam menos mão de obra e garantem rapidez na entrega.

\section{Conclusão}

Este artigo teve como objetivo analisar o comportamento institucional do ISI Eletrotécnica frente à perspectiva de busca de inovação a partir do relacionamento com empresas. Para isso foi feito um levantamento histórico das políticas industriais no Brasil e verificou-se que estas tiveram vários estágios. O mais importante para o objetivo deste artigo foi verificar que o último período de investimento em ações pró indústria aconteceu após 2003 e um dos resultados colhidos partir de 2013 foi a construção de uma rede de laboratórios de Inovação espalhados pelos diversos estados do país que são os Institutos SENAI de Inovação (ISI), no âmbito da EMBRAPII. Dessa rede, o ISI Eletroquímica foi o primeiro laboratório a entrar em funcionamento.

Considerando que o ISI é um instituto de pesquisa (IP) e busca interação como setor produtivo, foi necessário fazer uma análise do comportamento geral dos IPs no Brasil e no Paraná e seu relacionamento com empresas para logo analisar o caso das características do processo interativo especifico do ISI Eletrotécnica, (Paraná). Sobre a analise dos dados no DGP o que se conclui é:

- Apesar da quantidade total de IPs em geral (com e sem relacionamento) ser menor do que a quantidade de Us, em termos relativos, os IPs se relacionam mais do que as Us. A densidade de relacionamento dos IPs é maior do que das Us. Isso se explica pela necessidade de custeamento das suas ações que em geral são orientadas pela demanda.

- Os IP têm mais relacionamentos do tipo Rel2 - Pesquisa científica com considerações de uso imediato dos resultados e Rel7 - Transferência de tecnologia desenvolvida pelo grupo para o parceiro o que indica que atuam mais na perspectiva de soluções imediatas por conta da necessidade se obter rentabilidade. No Paraná o comportamento dos IPs é bastante similar.

Tendo em conta esse comportamento dos IPs foi utilizada uma ferramenta do ISI para prospecção de negócios e observou-se que o item econômico-financeiro detém $60 \%$ da importância de prospecção de um projeto, e posteriormente, são analisadas questões de rota tecnológica, parcerias, inovação, entre outros itens. Sendo assim, ainda que sua missão seja desenvolver pesquisa aplicada e acelerar a inovação tecnológica, suas decisões estão basicamente condicionadas aos determinantes de natureza econômico-financeira. 
96 A dinâmica de interação institutos de pesquisas/empresas: os condicionantes pró inovação do Instituto Senai de Inovação (ISI) Eletrotécnica

Essas decisões podem levar o ISI a se tornar mais uma organização que atende as empresas orientada por algumas demandas rentáveis e não necessariamente que busca desenvolver inovação com graus de ineditismo aplicado. Com escolhas voltadas a aceitar baixa relevância inovativa, projetos de baixa complexidade, corre-se o risco de que sua missão de promover inovação seja suplantada pela necessidade de se manter financeiramente estável.

Os dados do DGP indicaram também que as Us demonstram mais liberdade em termos de áreas do conhecimento em que atuam. Ou seja, existem muito mais GPs espalhados em muito mais áreas do conhecimento, ao contrário dos IPs que focam em áreas que certamente são mais promissoras (dentro de alguma perspectiva) ou cujas competências técnicas estão mais disponíveis. Nesse sentido, as universidades teriam sido também um instrumento importante de apoio à $\mathrm{P} \& \mathrm{D}$ a empresas dentro dessa perspectiva de política industrial propagada a partir da EMBRAPII. Por outro lado, como se mostrou aqui o ISI se encontra amplamente condicionado a determinante financeiro que reduzem o ritmo em que a inovação pode ser espalhada pelas empresas. A sua autonomia de atuação como agente de produção de inovação e desenvolvimento vai levar um tempo definido pela sua capacidade estratégica de atuar no mercado e conseguir atrair empresas para os tipos de desenvolvimentos possíveis no seu laboratório.

Por último, considerando que em geral os IPs no Brasil também são instituições autônomas, de natureza privada, sem fins lucrativos e que, portanto, trabalham com ampla interação com empresas, mas na perspectiva de auto sustentação, pode-se acreditar que os elementos aqui pesquisados podem ser mais ou menos replicados para os demais IPs dos país. Ou em outros termos, mais ou menos a mesma situação ocorre em IPs com as mesmas características.

\begin{abstract}
The Industrial, Technological, and Foreign Trade Policy (PITCE) from 2003 marks the resumption of the industrial policy after nearly 30 years of its absence. Other policies continued to leverage the innovative industrial production in the country. Among the various strategies, one of them was the construction of a national laboratory network to serve the industrial sectors in search of innovative solutions. It is the Senai Institute of Innovation (ISI). This article analyses the behaviour of the Research Institutes (IPs) from viewpoint of the characteristics and actions of the ISI Electrochemistry located in Curitiba, Paraná. As a methodology, we first observed the behaviour of the IPs based on the CNPq Research Group Directory data. The decision matrix of the ISI Electrochemistry was investigated. As a result, it is noticeable that more than innovative or unpublished research, the important thing is that the IP/enterprise relation must fundamentally be profitable for the IP. In other words, the focus on financial sustainability may lead institutes in general to be more profitable-demand-oriented than innovations-oriented with degrees of applied innovation.
\end{abstract}

Keywords: Innovation, Industrial Relationship Policy, Senai Institute of Innovation (ISI), universityenterprise 
JEL: L38, L52, O25

\section{Referências bibliográficas}

BRASIL, G. F. ESTRATÉGIA NACIONAL DE CIÊNCIA, TECNOLOGIA E INOVAÇÃO 2012 2015. Brasília, 2011. 220 p.

BRASIL, G. F. Plano Brasil Maior. Brasília, 2011. Disponível em: <http: //www.brasilmaior.mdic.gov.br/wpcontent/uploads/cartilhal_brasilmaior.pdf>.

CNPQ, C. N. d. P. C. e. T. Plano Tabular. 2017. Disponível em: <http://dgp.cnpq.br/ planotabular/index.jsp>.

DIAS, R. d. B. A trajetoria da política cientifica e tecnologica brasileira : um olhar a partir da analise de politica. 237 p. Tese (Doutorado) - Unicamp, 2009. Disponível em: <http://repositorio.unicamp.br/jspui/handle/REPOSIP/286686>.

EMBRAPII, A. B. d. P. e. I. I. Quem Somos. 2015. Disponível em: <http: //www.embrapii.org.br/categoria/institucional/quem-somos/>.

LIMA, P. G. Política Científica e Tecnológica: Países Desenvolvidos, América Latina e Brasil. Dourados, MS: Editora da UFGD, 2009. 169 p. ISBN 978-85-61228-55-2.

SCHWARTZMAN, S. Ciência e Tecnologia na década perdida: o que aprendemos? In: PAULANI, L. M.; SOLA, L. (Ed.). Lições da Década de 80. São Paulo, SP: EDUSP e UNRISD, 1995. p. 241-266.

SENAI, S. N. d. A. I. Instituto Senai de Inovação. 2015. Disponível em: <http: //www.portaldaindustria.com.br/senai/iniciativas/programas/inovacao-e-tecnologia/ institutos-de-inovacao/2014/09/1,46743/institutos-senai-de-inovacao.html>.

SUZIGAN, W.; ALBUQUERQUE, E. d. M. e.; CARIO, S. A. F. Em busca da inovacao : interacao universidade-empresa no Brasil. Belo Horizonte: Autêntica Editora, 2011. 463 p. ISBN 8575265822. 